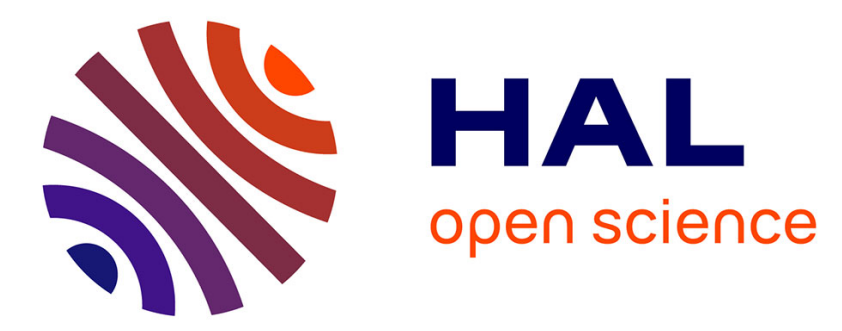

\title{
The effect of geometric and electric constraints on the performance of polymer-stabilized cholesteric liquid crystals with a double-handed circularly polarized light reflection band
}

\author{
Sabrina Relaix, Michel Mitov
}

\section{To cite this version:}

Sabrina Relaix, Michel Mitov. The effect of geometric and electric constraints on the performance of polymer-stabilized cholesteric liquid crystals with a double-handed circularly polarized light reflection band. Journal of Applied Physics, 2008, 104 (3), pp.033539. 10.1063/1.2968245 . hal-02887327

\section{HAL Id: hal-02887327 \\ https://hal.science/hal-02887327}

Submitted on 2 Jul 2020

HAL is a multi-disciplinary open access archive for the deposit and dissemination of scientific research documents, whether they are published or not. The documents may come from teaching and research institutions in France or abroad, or from public or private research centers.
L'archive ouverte pluridisciplinaire HAL, est destinée au dépôt et à la diffusion de documents scientifiques de niveau recherche, publiés ou non, émanant des établissements d'enseignement et de recherche français ou étrangers, des laboratoires publics ou privés. 


\title{
The effect of geometric and electric constraints on the performance of polymer-stabilized cholesteric liquid crystals with a double-handed circularly polarized light reflection band
}

\author{
Sabrina Relaix ${ }^{a)}$ and Michel Mitov ${ }^{\text {b) }}$ \\ Centre d'Elaboration de Matériaux et d'Etudes Structurales, CEMES, CNRS, Univ. Toulouse, BP 94347, \\ F-31055 Toulouse Cedex 4, France
}

(Received 4 April 2008; accepted 12 June 2008; published online 13 August 2008)

\begin{abstract}
Polymer-stabilized cholesteric liquid crystals (PSCLCs) with a double-handed circularly polarized reflection band are fabricated. The geometric and electric constraints appear to be relevant parameters in obtaining a single-layer CLC structure with a clear-cut double-handed circularly polarized reflection band since light scattering phenomena can alter the reflection properties when the PSCLC is cooled from the elaboration temperature to the operating one. A compromise needs to be found between the LC molecule populations, which are bound to the polymer network due to strong surface effects or not. Besides, a monodomain texture is preserved if the PSCLC is subjected to an electric field at the same time as the thermal process intrinsic to the elaboration process. As a consequence, the light scattering is reduced and both kinds of circularly polarized reflected light beams are put in evidence. Related potential applications are smart reflective windows for the solar light management or reflective polarizer-free displays with higher brightness. (C) 2008 American Institute of Physics. [DOI: 10.1063/1.2968245]
\end{abstract}

\section{INTRODUCTION}

Polymer-stabilized liquid crystals (PSLCs) are twocomponent composite materials made by photopolymerization and photocrosslinking of liquid crystalline monomers dissolved in a low molecular weight LC. ${ }^{1}$ As the reaction proceeds, the reactive material tends to phase separate from the LC, leading to the formation of a polymer network with a very high surface area. The cholesteric LC (CLC) helical structure results from macroscopic rotation caused by molecular chirality. When aligned into the so-called planar texture, it is distinguished by Bragg reflection of a circularly polarized light beam of the same handedness as the helix. ${ }^{2,3}$ Both the central position $\lambda_{0}$ and the width $\Delta \lambda$ of the reflection band are determined by the pitch $p$ of the helical structure, $\Delta \lambda=\left(n_{\|}-n_{\perp}\right) p$ and $\lambda_{0}=n p$, where $n_{\|}$and $n_{\perp}$ are the local values of the refractive indices of the CLC and $n=\left(n_{\|}\right.$ $\left.+n_{\perp}\right) / 2$ is the average refractive index. Modulating the characteristics of the reflection band (tuning $\lambda_{0}$ in the visible or infrared spectrum, broadening $\Delta \lambda$, or increasing the reflected light flux) is a practical goal that has driven numerous research efforts related to applications such as polarizer-free reflective displays ${ }^{4}$ (with no backlight requirement), polarizers and color filters, ${ }^{5}$ mirrorless lasing, ${ }^{6}$ or smart switchable reflective windows for the dynamical control of solar light. ${ }^{7}$ Since circularly polarized light of only one handedness is reflected by a CLC structure, the maximum reflection of ambient (unpolarized) light from a single-layer CLC is never greater than $50 \%$ at normal incidence. We have shown that this theoretical limit may be exceeded in a PSCLC singlelayer material. ${ }^{8,9}$ A low molecular weight CLC, which exhib-

\footnotetext{
${ }^{\text {a)} E l e c t r o n i c ~ m a i l: ~ s r e l a i x ~ @ 1 c i . k e n t . e d u . ~}$

${ }^{b)}$ Electronic mail: mitov@cemes.fr.
}

its a thermally induced inversion of helicity at the temperature $T_{C}$, was blended with nematic diacrylate monomers. The blend was then cured with ultraviolet (UV) light at a temperature $T^{+}>T_{C}$ when the helix was right handed. Due to the memory effects brought by the polymer network, more than $50 \%$ of the unpolarized incident light was reflected by the PSCLC at a temperature $T^{-}<T_{C}$ assigned to a CLC phase with the same pitch but a left-handed sense before reaction. The purpose of this paper, based on a different LC blend, is to report the crucial role of the confinement of the low molar LC in the porous polymer to allow the coexistence of both circularly polarized light components in the reflection band. Besides, the application of an electric field to the PSCLC, which has a negative dielectric anisotropy, preserves the reflected light flux, and the measurement of both kinds of circularly polarized reflected light beam is made possible. Since CLCs are used as tunable bandpass filters, reflectors, polarizers, and temperature (or pressure) sensors, novel opportunities to fabricate hyper-reflective displays and to modulate the reflection over the whole light flux range in a single-layer material are offered.

\section{EXPERIMENT}

A CLC oligomer (Wacker Chemie Ltd.) is blended with the chiral low molecular weight LC $(S, S)$-EPHDBPE or 4-[(S,S)-2, 3-epoxyhexyloxy]-phenyl-4-(decyloxy)-benzoate (Sigma-Aldrich), which is the helix-inversion compound (HIC). The oligomer is a cyclic polysiloxane molecule with two types of mesogen as lateral chains: a chiral (cholesterol group bearing) mesogen and an achiral (biphenyl group bearing) mesogen. ${ }^{10}$ The pitch of the cholesteric phase, and as a consequence the associated reflection wavelength, depends on the number of chiral mesogens. Two types of oligomer were chosen: a photocrosslinkable oligomer, called RMR, 


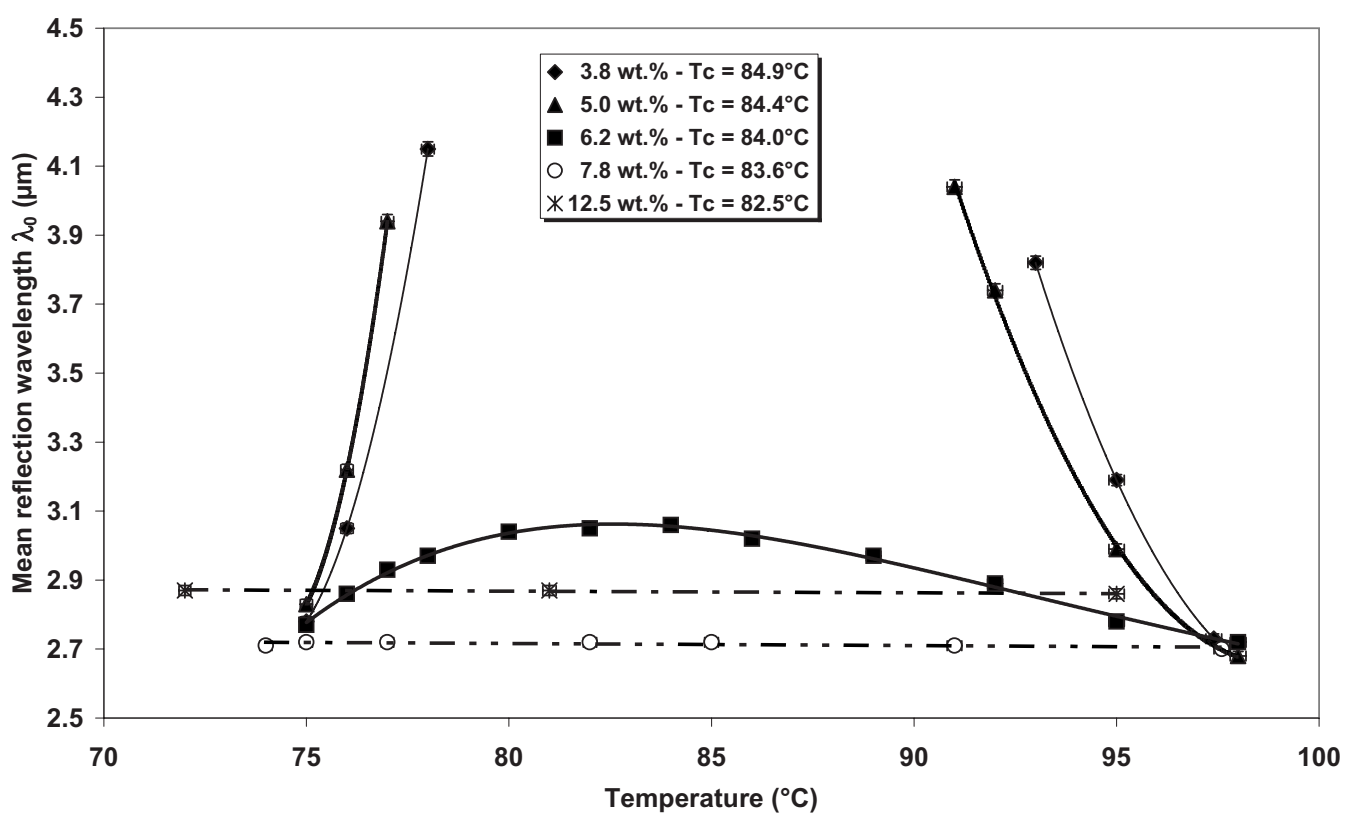

FIG. 1. Mean reflection wavelength of the PSCLC as a function of temperature for different monomer concentrations. The critical temperature $T_{C}$ at which the helicity inversion occurs is mentioned for each case.

and a nonphotocrosslinkable oligomer, called SR (both molecules have the same molar fraction of chiral mesogens, i.e., $31 \%$ ). RMR and SR were mixed together in different proportions to change the concentration in a network-forming material. This two-component mixture was blended with the $\mathrm{HIC}$ with a ratio constant for all the investigations and equal to $12.5: 87.5$ wt $\%$. With such an experimental protocol, it is possible to change the concentration in the network-forming material in the three-component mixture without drastically changing the pitch of the cholesteric phase. For each blend, 2 wt \% (compared to the RMR content) of photoinitiator Irgacure 907 (from Ciba-Geigy) is added for photocrosslinking purposes. The cholesteric structure is left (right) handed below (above) $T_{C}$.

The mixture was introduced at $89{ }^{\circ} \mathrm{C}$ by capillarity in a $25( \pm 5) \mu \mathrm{m}$ thick indium tin oxide (ITO)-coated glass cell. Before curing, the cell was kept at $T^{+}=98^{\circ} \mathrm{C}$ for a few minutes to favor the planar alignment of the blend. The sample was then irradiated with UV light $\left(0.1 \mathrm{~mW} / \mathrm{cm}^{2}\right.$ at $\left.365 \mathrm{~nm}\right)$ for $4 \mathrm{~h}$. Finally, the cell was cooled at $0.5{ }^{\circ} \mathrm{C} / \mathrm{min}$ until $T^{-}=75^{\circ} \mathrm{C}$.

The transmittance properties were investigated at normal incidence by infrared (IR) spectroscopy between 1.9 and $4.5 \mu \mathrm{m}$ with a Perkin-Elmer IR spectrometer spectrum 100. The baseline was made when the blend was in the nematic state at $T_{C}$. Measurements with circularly polarized incident light were performed using homemade circular polarizers fabricated by mixing two cyanobiphenyl CLC blends, BL094 and BL095 (from Merck Ltd.), for which the cholesteric phases exhibit the same pitch behavior but with opposite twist senses (BL094 and BL095 are enantiomers). The reflection wavelength as well as the twist sense of the cholesteric phase are tuned by varying their relative concentration: as a consequence, the kind of polarization is fixed. Due to the polarization selectivity rule for cholesteric reflectors, which are also polarizers, a left (right)-handed helix gives rise to a right (left) circular polarizer. When measurements with circularly polarized incident light were performed, a $25 \mu \mathrm{m}$ thick cell of pure BL094 (nonreflecting in the IR spectrum) is positioned between the source and the cell during the baseline step.

\section{RESULTS AND DISCUSSION}

\section{A. Effect of the polymer network concentration on the temperature dependence of the reflection wavelength}

The concentration in a network-forming material may determine the density of the network and the respective distribution of free and bound fractions of LC molecules in the volume of the PSLC. Here the RMR concentration in the two-component blend ranges from 3.8 to $12.5 \mathrm{wt} \%$. Figure 1 shows the variation in the selective reflection wavelength $\lambda_{0}$ of the PSCLC as a function of temperature for the different RMR concentrations.

When the RMR concentration is low, between 3.8 and $5.0 \mathrm{wt} \%$, the temperature behavior of $\lambda_{0}$ below as well as above $T_{C}$ is comparable to the behavior before curing; the influence of the polymer on the reflection properties is very weak. The pitch changes are hindered by the presence of oligomer molecules if the network has no tridimensional nature, and this situation is promoted for low concentrations. In that case, the well-aligned monodomain planar texture is broken, and light scattering occurs, especially when the temperature is below $T_{C}$. When the RMR concentration is between 7.8 and $12.5 \mathrm{wt} \%$, the $\lambda_{0}$ becomes independent of the temperature and is kept between 2.7 and $2.9 \mu \mathrm{m}$. The helical structure present at $T^{+}$is frozen into the composite material. The LC component undergoes strong surface interactions with the polymer network and has to be considered a bound fraction; the network is so dense that it hinders the reorganization of LC molecules as the temperature is lowered. Mid- 
way between the two previously described behaviors, the case of a concentration such as $6.2 \mathrm{wt} \%$ is especially interesting, and we are going to focus the rest of the paper on this case. The $\lambda_{0}$ versus $T$ curve is now bell shaped, which appears like the signature of an original intermediate memory effect, between the behaviors of the free fraction and the fully bound fraction. Indeed, a fraction of LC molecules behaves as free molecules since there is a variation in the position of the Bragg band with temperature: nevertheless this freedom is relative because $\lambda_{0}$ has limited values at each temperature. The divergence is even not attained inside the temperature range around $T_{C}$. In other words, the PSCLC material is still a reflector when its temperature is inside the temperature region in which the pitch diverged before curing. The reflection behavior is dominated by the structural changes of LC molecules that might be considered as an interfacial population between the completely free and bound populations of LC molecules. The $\lambda_{0}$ slightly increases from $2.7 \mu \mathrm{m}$ at $T^{+}$to about $3.1 \mu \mathrm{m}$ at $84{ }^{\circ} \mathrm{C}$, a temperature that corresponds to the top of the bell-shaped curve. Below this value, the decrease in $\lambda_{0}$, up to $2.7 \mu \mathrm{m}$ again at $T^{-}$, can be suspected of being the sign of a left-handed helicity, which is introduced in the structure of (at least a fraction of) LC molecules. This can indirectly be put in evidence by transmittance measurements under circularly polarized incident light; the related investigations are reported in Sec. III B.

\section{B. Impact of an electric field on the characteristics of the reflection band}

When a thermal ramp starts from $T^{+}$toward $T^{-}$, the CLC structure of the free fraction undergoes very drastic pitch changes, which even include unwinding of the helix at $T_{C}$ followed then by rewinding. Besides, these changes happen when the LC is strongly confined in a porous structure with a very large surface area. As a consequence, the formation of a well-aligned monodomain planar structure, which dominates at $T^{+}$, is not possible anymore and the CLC slab has a tendency to spontaneously produce a polydomain texture when it is cooled. A phenomenon of light scattering arises from this situation, which we have discussed in a previous paper; ${ }^{11}$ the slower the cooling rate, the smaller the transmission losses are. The light scattering phenomenon is more present in the material of the present study than in the previous one, ${ }^{11}$ in particular, because the confinement of LC molecules in the network is different. The confinement characteristics are mainly related to the nature of the polymer network: the flexibility of mesogens (of paramount importance), the concentration, the temperature, as well as the structure of the mesophase when the polymerization occurs. In close relation with the physical parameters of the blend, we propose here a solution to decrease the transmittance losses, which is an alternative to the decrease in the thermal ramp speed from $T^{+}$to $T^{-}$. Additionally, since measurements of circularly polarized light with both helicities and their relative distributions are desired, it is required to reduce or minimize the light scattering phenomenon and to preserve a planarly well-oriented texture. This objective was achieved by subjecting the cell to an electric field when the temperature was decreased from $T^{+}$to $T^{-}$and by taking advantage of
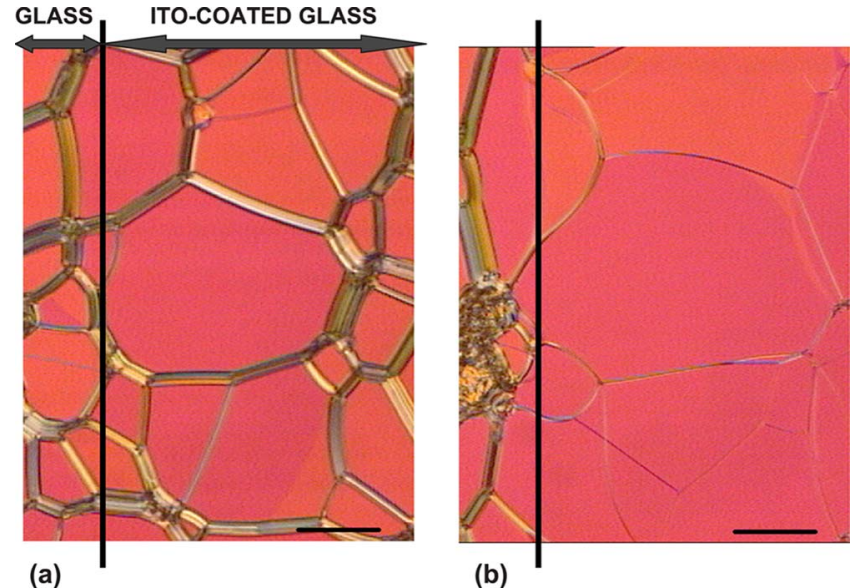

FIG. 2. (Color online) Optical micrographs of the cholesteric planar texture at the interface between the nonconducting and the (conducting) ITO-coated regions of the glass substrates at $T^{-}=74{ }^{\circ} \mathrm{C}$ after thermal process: (a) without electric field and (b) with an electric field (50 V at $1 \mathrm{kHz})$. Transmission mode. Polarizers are crossed. Scale bar $=100 \mu \mathrm{m}$.

the negative dielectric anisotropy $\Delta \varepsilon$ of the HIC. Indeed, an electric field applied to a CLC with a negative $\Delta \varepsilon$ and in a direction parallel to the helix axis stabilizes and favors a homogeneous planar texture because it tends to align the small axis of the molecules in the direction of the field (if $\Delta \varepsilon$ was positive, the helix would be progressively unwound). ${ }^{12}$ Therefore, a monodomain texture is favored to the detriment of a polydomain one and, in return, the scattering of the light is not promoted. For illustration, Fig. 2 shows the optical micrographs of the cholesteric planar texture at the interface between the nonconducting and the (conducting) ITO-coated regions of the cell glass substrates, with and without field. The texture exhibits oily streak defects, which can be considered like a network of defect lines dispersed in regions with a uniform helix direction; ${ }^{13}$ the structure of an oily streak mainly depends on elasticity and surface anchoring. Different red tints correspond to different twisted states (the number of pitch lengths varies from one region to the other one) due to hysteresis in temperature variation. It appears that the density and the thickness of oily streaks are greatly reduced when the CLC slab is subjected to an electric field. The network of oily streaks, which is generally not a static feature, is also modified very close to the interface between the nonconducting and conducting regions due to the edge effects.

Figure 3 shows the variation in the transmitted light losses due to the scattering as a function of temperature when an electric field $(91.5 \mathrm{~V}$ at $50 \mathrm{kHz})$ is applied or not. The losses are defined as the difference in light intensity transmitted by the PSCLC when measured out of the Bragg band at a wavelength arbitrarily chosen equal to $2 \mu \mathrm{m}$, at $T^{+}$, and at each measurement temperature $T$, i.e., $I^{2 \mu \mathrm{m}}\left(T^{+}\right)$ $-I^{2} \mu \mathrm{m}(T)$.

The losses increase when the temperature decreases but are clearly reduced when the electric field is applied during the thermal process. At the end (at $T^{-}=75^{\circ} \mathrm{C}$ ), the losses are reduced by more than four times. The influence of the electric field on the depth of the Bragg peak was also investi- 


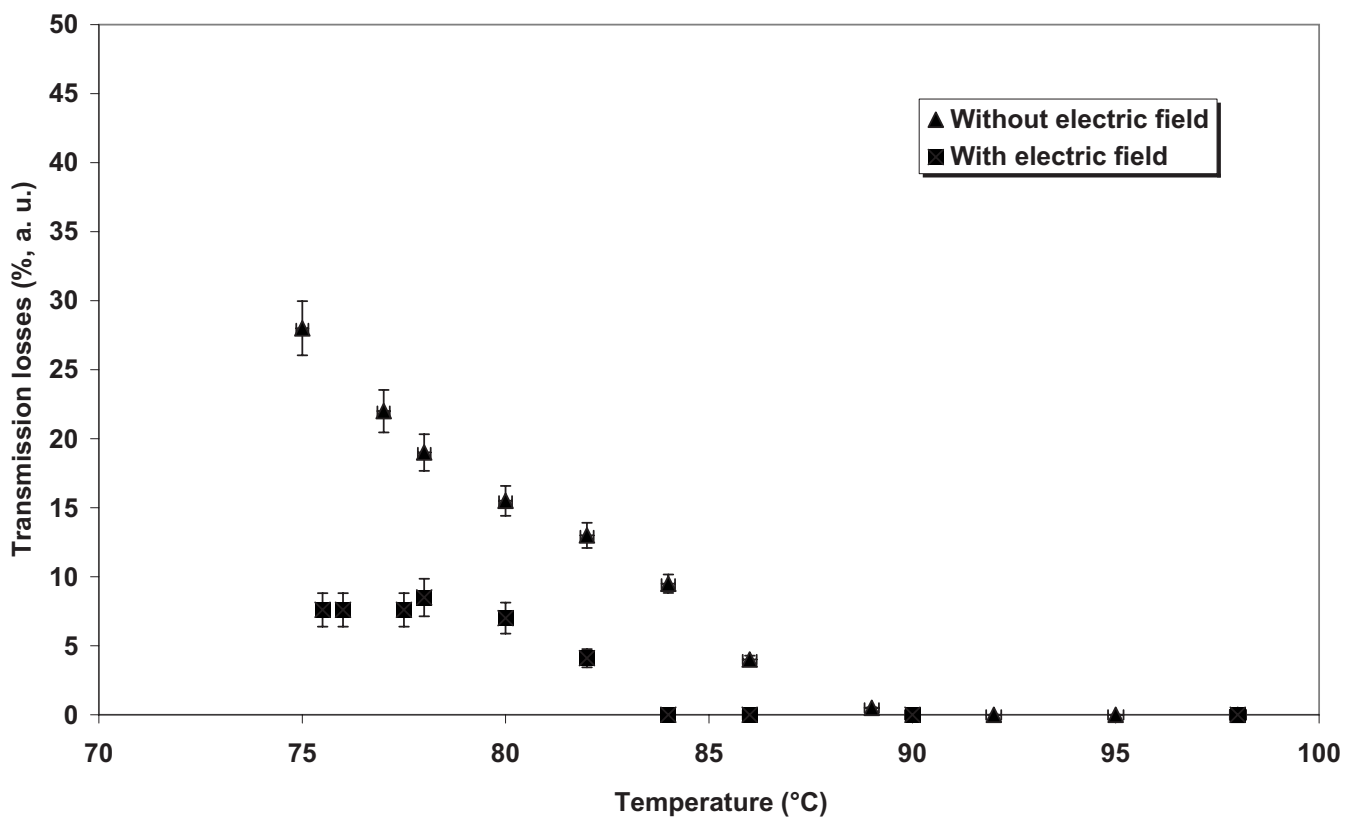

FIG. 3. Transmission losses due to light scattering as a function of temperature (monomer concentration=6.2 wt $\%$ ) when an electric field $(91.5 \mathrm{~V}$ at $50 \mathrm{kHz}$ ) is applied from $T^{+}$(curing temperature) to $T^{-}$(measurement temperature) or not.

gated. Figure 4 shows the variation in the reflected intensity as a function of temperature, with and without field.

When the material is not subjected to the field, the reflected light intensity progressively decreases by more than $10 \%$ when the temperature decreases. When the field is applied, the reflected intensity is preserved except in the temperature range between $T_{C}\left(=84{ }^{\circ} \mathrm{C}\right)$ and $77{ }^{\circ} \mathrm{C}$. This result is due to the decrease in $\lambda_{0}$ below $84{ }^{\circ} \mathrm{C}$, as can be seen in Fig. 1, and the introduction of the left helicity in the cholesteric structure of at least a fraction of LC molecules. After the temperature is decreased below this range, the reflected light intensity is restored to a value that is approximately the same as that at the beginning of the thermal process. It is checked that the application of an electric field does not induce any $\lambda_{0}$ shift (Fig. 5).

Then, the material subjected to an electric field is kept at $T^{-}$for a few tens of minutes. We measured the reflected intensity when the incident beam was right- or left-handed circularly polarized and we found $76 \%$ and $53 \%$, respectively. However, these quantities consist of the intensity of the light as reflected by the experimental cell and also by the polarizer. After measurement of the intensity reflected by the right- and left-circular polarizers $(43 \%$ and $42 \%$, respectively), we finally found for the intensity reflected by the

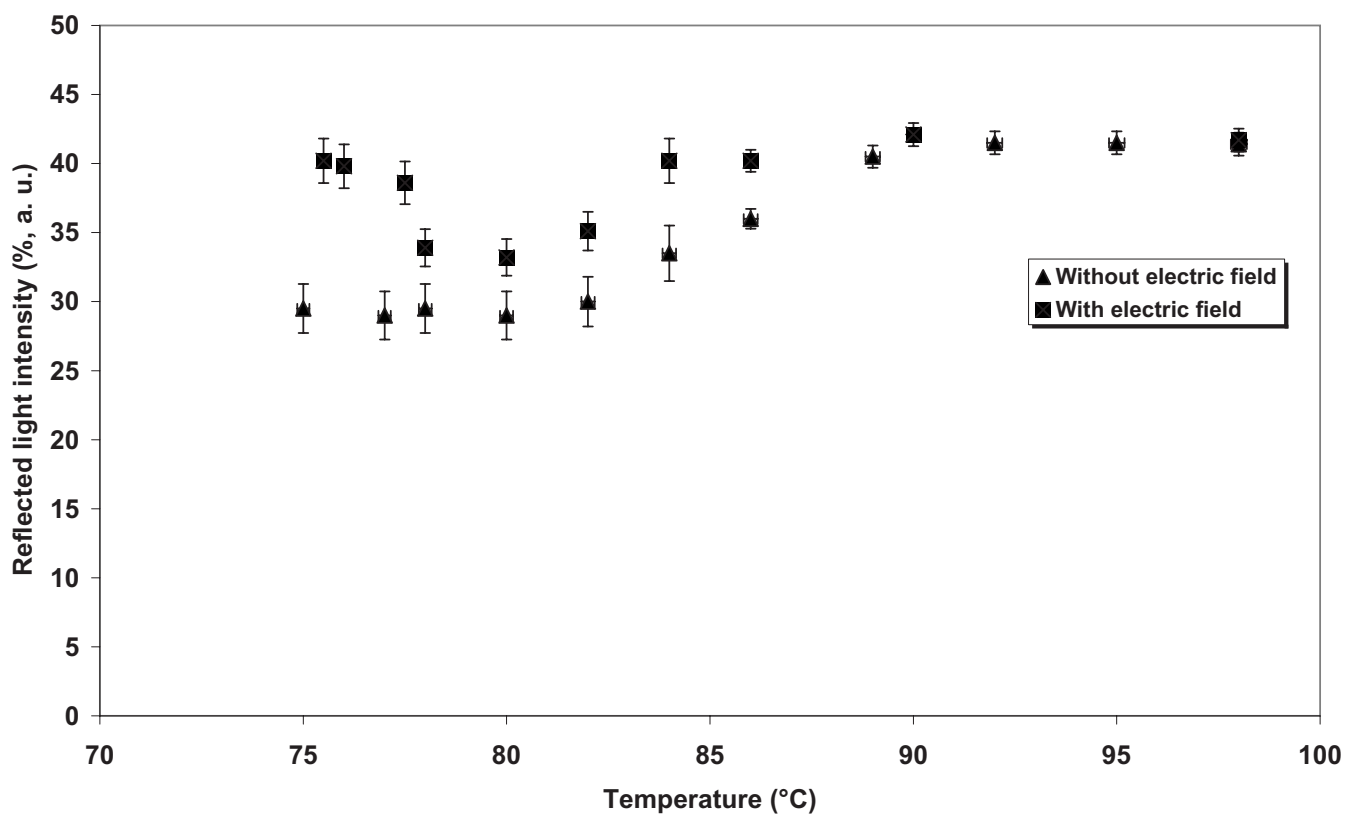

FIG. 4. Reflected light intensity as a function of temperature (monomer concentration $=6.2 \mathrm{wt} \%)$ when an electric field $(91.5 \mathrm{~V}$ at $50 \mathrm{kHz})$ is applied from $T^{+}$(curing temperature) to $T^{-}$(measurement temperature) or not. 


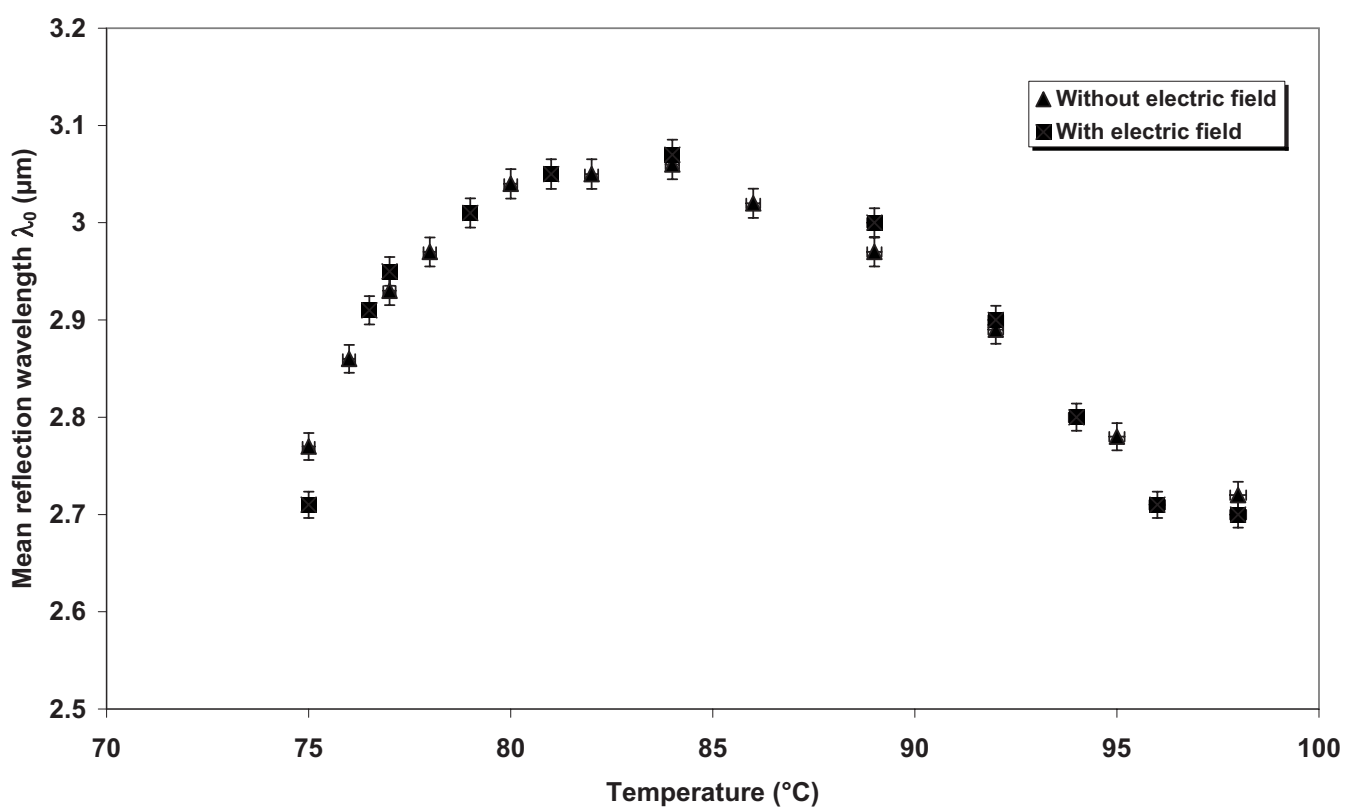

FIG. 5. Mean reflection wavelength as a function of temperature (monomer concentration $=6.2 \mathrm{wt} \%$ ) when an electric field $(91.5 \mathrm{~V}$ at $50 \mathrm{kHz})$ is applied from $T^{+}$(curing temperature) to $T^{-}$(measurement temperature) or not.

experimental cell when the incident beam is right or left circularly polarized the following values: $I_{R}=33 \%$ and $I_{L}$ $=11 \%$. When the electric field is switched off, the values are $I_{R}=29 \%$ and $I_{L}=7 \%$. This decrease shows that the texture relaxes toward a less homogeneously aligned planar texture.

In summary, the application of an electric field to the PSCLC when its temperature is changed from $T^{+}$to $T^{-}$allows (i) the reduction in the nucleation of a polydomain texture due to drastic pitch changes in the cholesteric structure of the free fraction by promoting a monodomain planar texture and (ii) the measurement of the reflected light intensity associated with the two distinct polarizations, which gives evidence to the property of double-handed circularly polarized light reflection. This property originates from the twopopulation structure of low molar mass LCs. Each population gives rise to a band of circularly polarized light that is selectively reflected. One was influenced to a large extent by the network and participated in the reflection of right-handed circularly polarized light, which corresponds to the reflectivity of the blend when the UV light curing occurred. The second population behaves much the same as in the bulk and participated in the reflection of left-handed circularly polarized light, which corresponds to the reflectivity of the blend at the final measurement temperature.

The procedure represents a technological alternative solution to the multilayer systems. Indeed, it is well known that two CLC cells with the same mean reflection wavelengths but opposite helicity senses may be stacked to increase the reflected light intensity. ${ }^{14}$ The cuticle of the beetle Plusiotis Resplendens also selectively reflects light with a doublehanded circular polarization between 520 and $640 \mathrm{~nm} .{ }^{15}$ The chitin molecules of the three-layer cuticle adopt a liquid crystalline structure: a half-wave-plate, made of molecules with a nematic organization, is included between two cholesteric layers of left-handed helicity sense; the right-handed polarized light that is passed through the first layer is con- verted into left-handed polarized light by the half-wave-plate and finally reflected from the third layer. However, a multilayer solution is typically undesirable due to the optical losses at the interfaces (reflection, scattering) and the cost of implementation for applications.

\section{CONCLUSION}

A double-handed circularly polarized light band may be reflected by a PSCLC single-layer material. We have found the right balance between the free and bound populations of LC molecules, which is related to the concentration in a network-forming material and, finally, to the geometrical confinement of the LC into the network. Light scattering may arise from a polydomain structure due to the drastic pitch changes in the helical structure of the free population when the temperature is changed from the polymerization temperature to the measurement one. We have found a solution for preserving a monodomain texture and, in return, increasing the reflected light intensity by subjecting the PSCLC (which has a negative dielectric anisotropy) to an alternative electric field during the thermal process. Consequently, it was possible to discriminate the respective quantities of left and right circular polarized components in the total amount of reflected light intensity. Potential applications are related to the light management for smart windows or reflective polarizer-free displays with a larger scale of reflectivity levels and, more generally, for regulation of temperature, telecommunications, or stealthiness.

${ }^{1}$ Liquid Crystals in Complex Geometries, edited by G. P. Crawford and S. Zumer (Taylor \& Francis, London, 1996).

${ }^{2}$ P.-G. de Gennes and J. Prost, The Physics of Liquid Crystals (Oxford University Press, Oxford, 1993), pp. 264-268.

${ }^{3}$ M. Mitov, Les Cristaux Liquides (Presses Universitaires de France, Paris, 2000), pp. 44-49.

${ }^{4}$ Y. Huang, Y. Zhou, and S.-T. Wu, Opt. Express 15, 6414 (2007)

${ }^{5}$ N. Y. Ha et al., Nat. Mater. 7, 43 (2008). 
${ }^{6}$ W. Cao, A. Munoz, P. Palffy-Muhoray, and B. Taheri, Nat. Mater. 1, 111 (2002).

${ }^{7}$ M. Mitov, E. Nouvet, and N. Dessaud, Eur. Phys. J. E 15, 413 (2004).

${ }^{8}$ M. Mitov and N. Dessaud, Nat. Mater. 5, 361 (2006).

${ }^{9}$ M. Mitov and N. Dessaud, C.R. Acad. Sci., Ser. IIc: Chim 11, 253 (2008)

${ }^{10}$ F.-H. Kreuzer, N. Häberle, H. Leigeber, R. Maurer, J. Stohrer, and J. Weis, in Organosilicon Chemistry III, edited by N. Auner and J. Weis (Wiley,
New York, 1997), pp. 566-586.

${ }^{11}$ M. Mitov and N. Dessaud, Liq. Cryst. 34, 183 (2007).

${ }^{12}$ L. M. Blinov and V. G. Chigrinov, Electrooptic Effects in Liquid Crystal Materials (Springer-Verlag, New York, 1994), pp. 319-323.

${ }^{13}$ I. Dierking, Textures of Liquid Crystals (Wiley, Weinheim, 2003).

${ }^{14}$ D. M. Makow, Appl. Opt. 19, 1274 (1980).

${ }^{15}$ S. Caveney, Proc. R. Soc. London, Ser. B 178, 205 (1971). 